\title{
Prevalence of thermophilic Campylobacter species in Swedish dogs and characterization of $C$. jejuni isolates
}

\author{
Mia Holmberg ${ }^{1 *}$, Thomas Rosendal ${ }^{1}$, Eva O Engvall ${ }^{2}$, Anna Ohlson ${ }^{3}$ and Ann Lindberg ${ }^{1}$
}

\begin{abstract}
Background: The aims of this study were to investigate the prevalence of Campylobacter species in Swedish dogs, to identify the species of the Campylobacter isolates and to genotype the C. jejuni isolates. Young and healthy dogs were targeted and the sampling was performed at 11 veterinary clinics throughout Sweden from October 2011 to October 2012. Faecal swab samples were collected and sent to the laboratory at the National Veterinary Institute (SVA) for isolation of Campylobacter, speciation and genotyping.

Results: Campylobacter spp. were isolated from 67 of the 180 sampled dogs which yields an overall prevalence of $37 \%$. The most prevalent species of Campylobacter among the participating dogs was C. upsaliensis with 52 of the 67 identified isolates. A lower prevalence was observed for C. jejuni with seven identified isolates and one isolate was identified as C. helveticus. Multi-locus sequence typing (MLST) was carried out on the seven C. jejuni isolates and all sequence types that were found are also commonly found in humans. The dogs were divided into three age groups; 1) under 12 months, 2) 12 to 23 months and 3) 24 months and older. The highest prevalence was found in the two younger age groups. Dogs shedding C. jejuni were between 3-12 months of age while dogs shedding C. upsaliensis were found in all ages.

Conclusions: The present investigation finds that Campylobacter spp. known to cause campylobacteriosis in humans are present in Swedish dogs. The results suggest an age predisposition where dogs under 2 years of age are more likely to shed Campylobacter spp. than older dogs. The most commonly isolated species was C. upsaliensis followed by C. jejuni, which was only detected in dogs up to 12 months of age. All C. jejuni isolates identified in the present study were of the same MLST types that have previously been described both in humans and in animals. The awareness of the Campylobacter risk of healthy young dogs may be an important way to reduce the transmission from dogs to infants, young children and immunocompromised adults.
\end{abstract}

Keywords: Dog, Campylobacter, C. upsaliensis, C. jejuni, MLST, Prevalence

\section{Background}

Campylobacteriosis is the most commonly reported zoonotic disease and the most common cause of bacterial enteritis in humans in many countries throughout the world [1]. In 2013, there were 8114 notified human cases of campylobacteriosis in Sweden. Of these, $41 \%$ were considered to be domestically acquired [2]. Humans can be infected with Campylobacter by several routes and the bacteria are commonly found in a wide range of

\footnotetext{
* Correspondence: mia.holmberg@sva.se

1 Department of Epidemiology and Disease Control, National Veterinary Institute, SVA, SE-751 89 Uppsala, Sweden

Full list of author information is available at the end of the article
}

animals including cats and dogs. Especially in young dogs ( $<1$ year), Campylobacter is often found in faecal samples and the dogs usually shed the bacteria without showing any clinical signs $[3,4]$. Several studies have reported the presence of Campylobacter spp. in both healthy dogs and dogs with diarrhoea, but Campylobacter is generally not considered to be pathogenic to dogs [5-8]. No association was found between presence of Campylobacter and diarrhoea in a Norwegian study on dogs [8] whereas a study in Canada found significant higher infection rate in diarrhoeic dogs compared with healthy dogs [5]. In an investigation of dogs in Ireland, diarrhoeic dogs were more likely to be Campylobacter positive than dogs without 
diarrhoea, but the dogs with diarrhoea also had concurrent gastrointestinal parasites, inflammatory bowel disease or diabetes [9]. A study of dogs in Barbados found no difference in clinical disease in dogs with and without presence of Campylobacter in the faeces, but indicated that co-infection with parvovirus and Campylobacter was common [10]. An association between occurrence of diarrhoea and Campylobacter infection was described in a previous Swedish investigation of dogs from 1979, and 63\% of the dogs with diarrhoea also had antibodies to canine parvovirus [11]. The true role of Campylobacter in canine gastroenteritis is uncertain as the bacteria may be found in clinically healthy dogs or often as a co-infection or intestinal carriage in diseased dogs.

In most studies, the predominant Campylobacter species isolated from dogs is C. upsaliensis and dogs are regarded as an important reservoir for this species $[3-6,9,12]$. The second most common Campylobacter species isolated from dogs, in many populations, is $C$. jejuni $[3,4,6,9,12]$, which is also responsible for the majority of human infections $[13,14]$. The reported prevalence of Campylobacter in dogs varies widely between studies, ranging from $22 \%$ to $100 \%$ and is reported to depend on factors such as the age, diet and housing of the dogs. Previous prevalence investigations of Campylobacter in dogs have also varied by study design and method of bacterial isolation.

Human campylobacteriosis is frequently attributed to contact with contaminated food (especially poultry meat) or water $[15,16]$. In several studies, direct contact with pet animals has also been identified as a possible source of human Campylobacter infection [15,17-19]. Presence of a puppy in the household has been identified as risk factor for campylobacteriosis, especially in young children [20,21]. In a study by Wolfs et al. [19] evidence was presented for transmission of $C$. jejuni from a dog to a 3-week old infant. However, a study by Studahl and Andersson [16] did not find a significant association between human campylobacteriosis and contact with dogs.

The present survey is part of an ongoing more comprehensive Campylobacter source attribution study in Sweden. Strains from human cases of campylobacteriosis were collected during the same time period as samples were taken from dogs, cattle, pigs, sheep, poultry and wild birds. Other relevant sources of human campylobacteriosis such as retail poultry meat, raw water and bathing water were also sampled during this period.

The aim of this study was to update our knowledge on the prevalence of Campylobacter spp. in young dogs in Sweden by collecting samples from healthy, young dogs throughout a year. The aim was also to identify the species of the Campylobacter isolates and to genotype the C. jejuni isolates by multi-locus sequence typing (MLST) to enable comparison between $C$. jejuni isolates from dogs and humans.

\section{Methods}

\section{Study population and sampling}

\section{Selection of clinics to participate in the study}

The sampling of dogs was performed at veterinary clinics throughout Sweden from October 2011 through October 2012. The geographical regions (counties) to be included in the survey were selected depending on the number of reported human cases of campylobacteriosis in recent years (based on data from the Public Health Agency of Sweden, www.folkhalsomyndigheten.se). The counties with the highest incidence of human campylobacteriosis were prioritised but the aim was also to cover a large part of the country. Requests for participation in the sampling process were sent to 53 veterinary clinics that had a previously established contact with the laboratory at Department of Bacteriology at the National Veterinary Institute (SVA, www.sva.se). A selection of 11 was made from the 18 veterinary clinics that had agreed upon participation in the study. One of them, located in the north of Sweden was included in the survey despite the lower incidence of human campylobacteriosis in favour of the better geographic coverage.

\section{Sampling procedure}

The requirements for dogs to be sampled were that they were under the age of two, weaned and healthy with no signs of diarrhoea. Young dogs were targeted because the prevalence of Campylobacter spp. is likely to be highest in young animals $[4,6,22]$. The aim was to collect a total of 200 samples in the study in order to obtain approximately 100 isolates. Based on a previous study in Sweden by Engvall et al. [4] an overall isolation rate of Campylobacter spp. of around 50\% was expected. The clinics were instructed to sample one to two dogs per month. Each dog was sampled only once during the sampling period. Faecal samples were collected from freshly voided faeces and sent to SVA on swabs in Amies transport medium with charcoal (Amies agar gel swabs - with charcoal, Copan, Italy). The swab samples were sent to SVA by ordinary mail at the day of sampling for isolation of Campylobacter, species identification and genetic subtyping of $C$. jejuni isolates. Written consent of the animal owners was obtained for sampling along with details about the age of the dog and postal code of the owner.

\section{Isolation and species identification of Campylobacter}

Swab samples were cultured on modified charcoal, cefoperazone, desoxycholate agar (mCCDA), (Oxoid Inc, Basingstoke, Hampshire, UK) and incubated at 41. $5 \pm$ $1.0^{\circ} \mathrm{C}$ for up to 5 days in a microaerobic atmosphere created by use of Campygen (Oxoid Inc) or Anoxomat (Advanced Instruments, Inc., Norwood, Massachusetts, USA). Preliminary identification of Campylobacter spp. was based on colony and microscopic morphology and 
the following phenotypic and biochemical characteristics and tests; motility, oxidase-, catalase-, hippurate and indoxyl acetate reactions. Strains confirmed as Campylobacter spp. were stored at $-80^{\circ} \mathrm{C}$ until further identification. Identification of $C$. jejuni was mainly based on a positive hippurate test. The species identification of all hippuratenegative isolates was done by mass spectrometry, time of flight, Maldi-Tof [23]. All isolates that were identified as C. upsaliensis by Maldi-Tof were further tested by polymerase chain reaction (PCR) for confirmation $[24,25]$.

\section{Genotyping of C. jejuni}

MLST was carried out as previously described by Dingle et al. [26] on the isolates identified as C. jejuni. Alleles, sequence type (ST) and clonal complex were assigned using the pubMLST database (http://pubmlst.org/campylobacter). Sequence types that shared four or more alleles were considered to belong to the same clonal complex. Because the present study is part of a larger comparative study of C. jejuni between various sources in Sweden, genotyping was restricted to this Campylobacter species.

\section{Results}

In total, 180 dogs were sampled from the 11 participating veterinary clinics. The sampling period was from October 2011 to October 2012; however, two samples received in November 2012 were also included in the study. For practical reasons, the number of samples received per month varied between the participating clinics. One clinic only sent in samples from one month. The location of the clinics and number of sampled and positive dogs per clinic is illustrated in Figure 1. One of the positive samples lacked information about clinic on the referral form. Most samples were received during the first seven months of the sampling period (October April). The highest proportion of positive samples occurred in the winter months with a peak in March 2012 (57\%). The number of samples per month and positive samples per month are shown in Figure 2.

The age distribution of the sampled dogs ranged from one month to 11 years. The aim was to sample dogs under the age of two, although 17 samples turned out to be from dogs between 2 and 11 years and 9 samples lacked information about age. The average and median age was 12 months. The dogs were divided into three age groups; 1) under 12 months, 2) 12 to 23 months and 3) 24 months and older. Number of samples and prevalence of Campylobacter in the different age groups are shown in Figure 3. Highest prevalence was found in the two younger age groups (37\% and $40 \%$ for group 1 and 2 , respectively) and the lowest in age group $3(12 \%)$.

Campylobacter spp. were isolated from 67 of the 180 sampled dogs which yields an overall prevalence of $37 \%$ in this material. The most common species of Campylobacter isolated from the dogs was C. upsaliensis with 52 of the 67 identified isolates (78\%). A lower occurrence of C. jejuni was observed, with 7 identified isolates (9\%) and 1 isolate (1\%) was identified as C. helveticus. All C. jejuni samples were found in dogs up to 12 months of age. In addition there were 7 isolates of Campylobacter species that did not survive the storage process, prior to the speciation. These isolates were all hippurate negative which indicates that they were most likely not $C$. jejuni, but could not be identified to species level by Maldi-Tof or PCR.

Results from the MLST are shown in Table 1. The dogs shedding $C$. jejuni were sampled at different locations except for two that were sampled in the same clinic. All seven isolates had different ST types. However, two dogs, sampled at different locations, were shedding C. jejuni of the same clonal complex, ST Clonal complex 21.

\section{Discussion}

In this study, $37 \%$ of the tested dogs were found to be positive for Campylobacter. This proportion is similar to the previous prevalence estimates of other studies $[6,9,27]$. However, as we targeted young dogs and the mean age of the sampled dogs was 12 months this estimated prevalence may not be representative for the entire Swedish dog population. Moreover, the study design was such that each dog was only sampled once and only one isolate per dog was analysed. In longitudinal studies with another type of study design where dogs were sampled at several occasions considerably higher prevalences of $73-100 \%$ have been reported $[3,28]$. The most common Campylobacter species among the tested dogs (52/180 dogs) was by far C. upsaliensis which is in agreement with many previous studies on dogs where relatively high prevalences of C. upsaliensis have been found in many different populations and countries [5,7,9,22,28]. The lower prevalence $(4 \%)$ that was observed for C. jejuni $(7 / 180$ dogs $)$ is similar to some studies $[12,27,29,30]$, but lower than in a previous Swedish study by Engvall et al. [4] where $11 \%$ of the dogs were positive for C. jejuni. However, in the previous study all samples were cultured on three selective plates (two CAT and one Preston agar plate). This probably contributed to the higher isolation rate compared to the present study. A recent study in Switzerland by Amar et al. [30] found a low prevalence of $C$. jejuni (6.3\%) and an even lower prevalence $(5.9 \%)$ of $C$. upsaliensis in healthy dogs. A higher prevalence of $C$. jejuni has been reported in stray dogs $[10,28]$, which may be due to a different exposure to environmental sources of Campylobacter than what household dogs are normally exposed to. In a study from Spain, 19\% (20/105) of dogs under 2 years of age living in a household, were found to have C. jejuni [22]. In the present study, one sample was identified as C. helveticus 


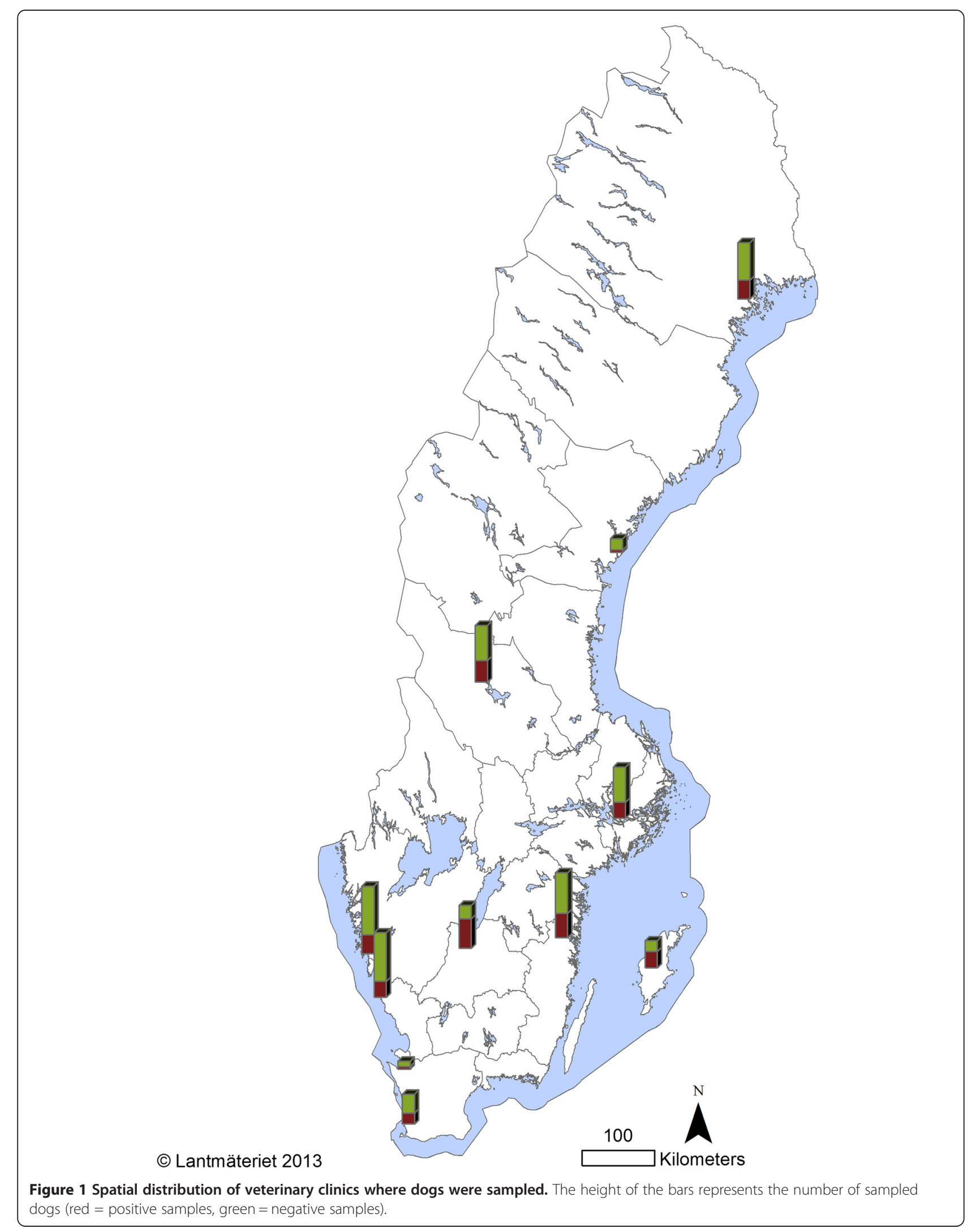




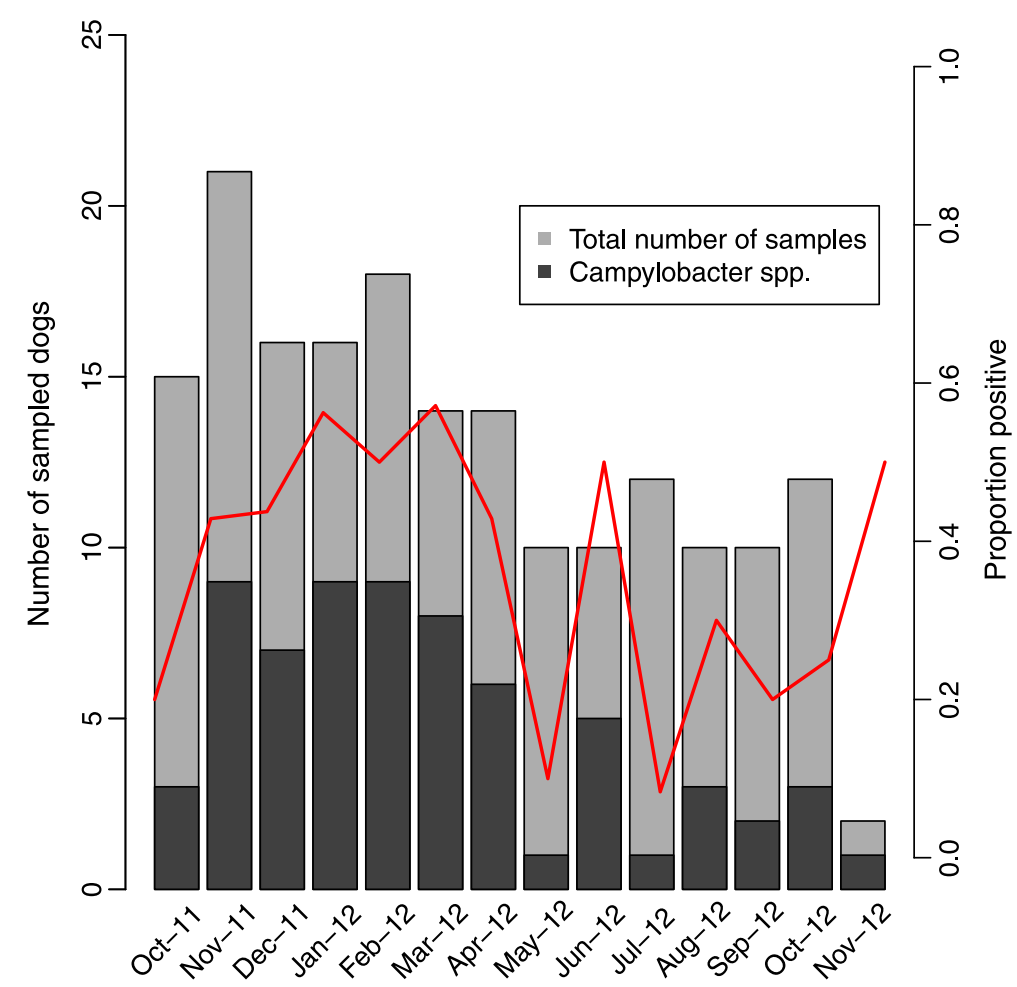

Figure 2 Total number of samples and samples with Campylobacter species per month. Proportion positive samples is indicated by the red line.

which is not so often identified in samples from dogs, but has previously been associated with cats [29].

Although C. upsaliensis is not isolated very frequently from humans in routine investigations it is possible that available data underestimate the prevalence due to the methods that are used at the public health laboratories which are primarily developed to detect $C$. jejuni and C. coli. In Sweden and many countries in Europe, human clinical Campylobacter isolates are not identified to species level which might contribute to a lack of data regarding the prevalence of C. upsaliensis in humans [31]. The role of $C$. upsaliensis in human disease is not very well established but it has been shown that $C$. upsaliensis can be a cause of gastroenteritis in both adults and children [32,33]. Labarca et al. [34] found that C. upsaliensis was the second most frequently isolated species in humans after $C$. jejuni. The authors also found that three dogs living in the households of two human patients infected with $C$. upsaliensis had the same Campylobacter species isolated in their stool specimens, but were not from the same clonal complex [34]. A study in Belgium reported that an outbreak in four day care centres was caused by C. upsaliensis [35]. Damborg et al. [36] found that a cluster of human C. upsaliensis strains was unrelated to dog strains of $C$. upsaliensis examined in the study by AFLP fingerprinting. However, the human and dog samples were not collected in the same countries which makes it difficult to interpret the results with regard to host specificity.

The age of each dog at sample collection was recorded and the dogs were divided into three age categories for comparison of prevalences. The results were in agreement with many other studies that have reported higher prevalence of Campylobacter in younger dogs or puppies compared with adult dogs $[6,9,12,22,27,37]$. These results suggest an age predisposition where young dogs are more susceptible to colonisation, possibly due to the development of immunity with age. Senior dogs have also been found to be at risk for Campylobacter colonisation [27,28]. Wieland et al. [29] found a significant association with age and $C$. upsaliensis but no association between age and presence of $C$. jejuni. Dogs shedding C. jejuni in the present study were all young, between 3-12 months while dogs shedding C. upsaliensis were found in all age categories, which is similar to what was described by Hald et al. [3].

Seasonality that is observed in human campylobacteriosis with peaks during the summer months [31] was not observed in this material as the highest incidence was observed in the winter months with a peak in March. Due to 


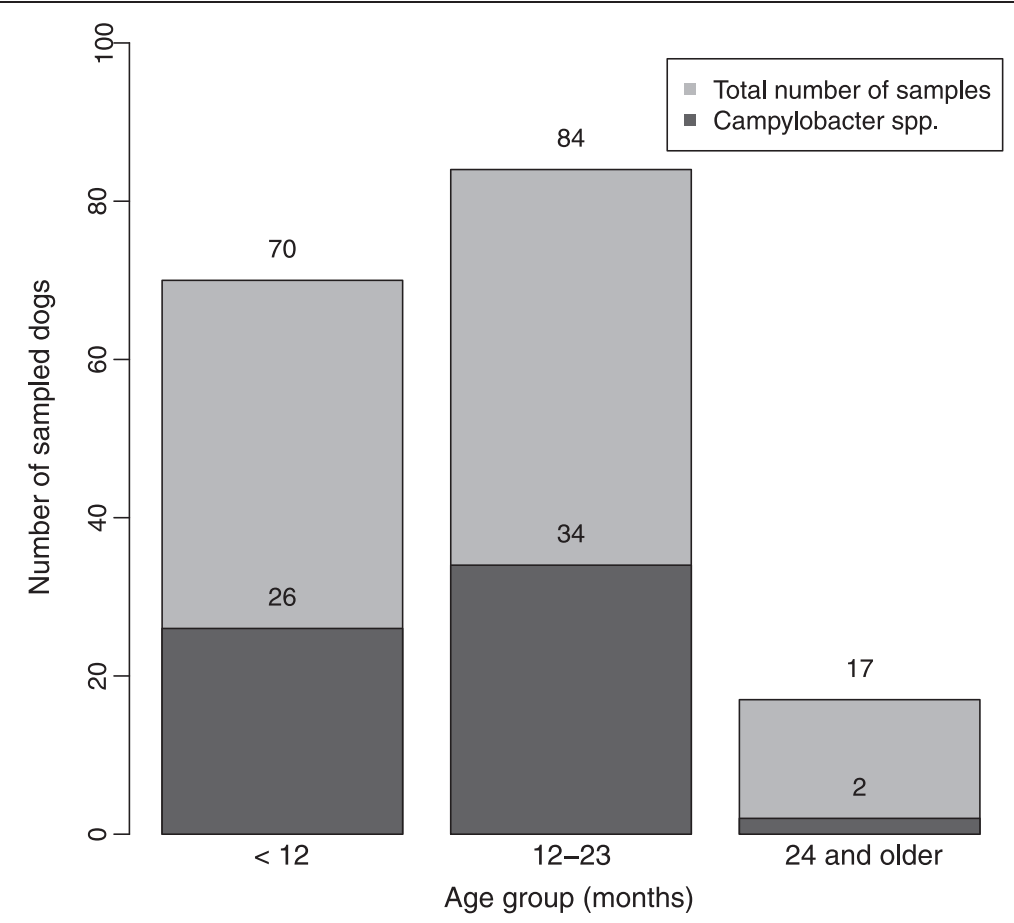

Figure 3 Number of dogs in different age groups that tested positive or negative for Campylobacter species.

the low number of samples per month it is not possible to draw any conclusions regarding seasonality based on our findings. Carbonero et al. [22] found a significantly higher prevalence of $C$. jejuni in dogs during spring compared to winter. They also reported a higher prevalence of C. upsaliensis during the summer. Hald et al. [3] did not find seasonal variation in carrier rates among dogs in a longitudinal study.

Molecular typing techniques enable comparison of sequence types (STs) between humans and the potential source of Campylobacter. In our study, seven C. jejuni samples were isolated and further subtyped by MLST. The results show high heterogeneity as all isolates were of different STs and only two isolates (from dogs sampled in different areas) belonged to the same clonal complex, ST21. Since only 7 C. jejuni isolates were identified and typed, the true extent of the $C$. jejuni population diversity in Swedish dogs cannot be estimated from this material. Mughini Gras et al. [21] showed a high degree of overlap between human and pet (dog and cat) C. jejuni STs. They identified two cases where identical $C$. jejuni STs (ST45 and ST658) were isolated from dogs and their owners. Four of the STs identified in the present study (ST50, ST45, ST257 and ST122) belong to those more frequently found STs in pet owners in the study by Mughini Gras et al. [21]. Manning et al. [38] found that a majority of the identified C. jejuni ST-complexes overlap between human and various animal sources. When comparing to the Campylobacter MLST database of C. jejuni isolates (http:// pubmlst.org/campylobacter) all seven STs that were found are common human STs. In the study by Amar et al. [30] the most frequent STs identified in dogs include ST45 and ST21 that was found also in the present study. Parsons et al. [28] also reported that ST45 was the most

Table 1 Results from multi-locus sequence typing (MLST) of Campylobacter isolates from dogs

\begin{tabular}{llllll}
\hline Dog & Age (months) & Location of veterinary clinic & County of Sweden & MLST & MLST-complex \\
\hline 1 & 12 & Västra Frölunda & Västra Götaland & 50 & ST-21 complex \\
2 & 4 & Jönköping & Jönköping & 883 & ST-21 complex \\
3 & 12 & Jönköping & Jönköping & 52 & ST-52 complex \\
4 & 4 & Ängelholm & Skåne & 45 & ST-45 complex \\
5 & 3 & Varberg & Halland & 122 & ST-206 complex \\
6 & 12 & Gamleby & Kalmar & 257 & ST-257 complex \\
7 & 4 & Gammelstad & Norrbotten & 677 & ST-677 complex \\
\hline
\end{tabular}


common complex in dogs at a rescue kennel. Many studies have identified ownership or close contact with dogs as a potential risk factor for human campylobacteriosis. However, despite the use of molecular information it is often difficult to know whether the bacteria is transferred from dog to human or human to dog or was acquired from the same common source.

In general, it is likely that dogs and humans are exposed to common sources of $C$. jejuni. The relatively low prevalence of $C$. jejuni in dogs in this study suggests that the importance of dogs for human infections compared with other sources such as food products may be low. However, dogs and in particular puppies are likely to have close contact with their owner and children in a family. Considering this it is important to note that all C. jejuni positive dogs were puppies or young dogs up to 12 months old. As the prevalence of $C$. upsaliensis in dogs under two years of age is relatively high there is a risk for transmission of zoonotic Campylobacter from dogs to humans and especially young children that are more susceptible for infection.

\section{Conclusion}

The present investigation finds that Campylobacter spp. known to cause campylobacteriosis in humans are present in Swedish dogs. The results suggest an age predisposition where dogs under 2 years of age are more likely to shed Campylobacter spp. than older dogs. The most commonly isolated species was $C$. upsaliensis followed by $C$. jejuni, which was only detected in dogs up to 12 months of age. All $C$. jejuni isolates identified in the present study were the same MLST types as had been previously described both in humans and in animals. Further investigation is necessary to determine the similarity between these dog C. jejuni MLST types and those found in humans in Sweden during the same period. The awareness of the Campylobacter risk of healthy young dogs may be an important way to reduce the transmission from dogs to infants, young children and immunocompromised adults.

\section{Competing interests}

The authors declare that they have no competing interests.

\section{Authors' contributions}

AL designed and coordinated the study, EE participated in the design of the study and coordinated the laboratory work, AO was responsible for selection of clinics and the sampling process, $\mathrm{MH}$ was the main author of the manuscript and performed the data analysis, TR participated in the data analysis and the preparation and editing of the manuscript. All authors have read and approved the final manuscript.

\section{Acknowledgements}

We are grateful to all dog owners who let their dogs be part of the study, we also gratefully acknowledge the veterinary clinics for their collaboration in the project. We would like to thank Mattias Myrenås, Boel Harbom and Ninni Pudas for their work in the lab with MLST, PCR and Maldi-tof. We would also like to thank the personnel at the Department of Bacteriology at the National Veterinary Institute for their work with analysing all the samples for presence of Campylobacter. This study was funded by the Swedish research council Formas.

\section{Author details}

'Department of Epidemiology and Disease Control, National Veterinary Institute, SVA, SE-751 89 Uppsala, Sweden. ${ }^{2}$ Department of Bacteriology, National Veterinary Institute, SVA, SE-751 89 Uppsala, Sweden. 'Växa Sverige, Box 30204, SE-104 25 Stockholm, Sweden.

Received: 2 October 2014 Accepted: 20 March 2015

Published online: 01 April 2015

\section{References}

1. EFSA, ECDC. The European Union Summary Report on Trends and Sources of Zoonoses, Zoonotic Agents and Food-borne Outbreaks in 2011. EFSA J. 2013;11(4):3129. doi:10.2903/j.efsa.2013.3129.

2. Folkhälsomyndigheten. http://folkhalsomyndigheten.se/amnesomraden/ statistik-och-undersokningar/sjukdomsstatistik/campylobacterinfektion/. 2014.

3. Hald B, Pedersen K, Waino M, Jorgensen JC, Madsen M. Longitudinal study of the excretion patterns of thermophilic Campylobacter spp. in young pet dogs in Denmark. J Clin Microbiol. 2004;42(5):2003-12.

4. Engvall EO, Brandstrom B, Andersson L, Baverud V, Trowald-Wigh G, Englund L. Isolation and identification of thermophilic Campylobacter species in faecal samples from Swedish dogs. Scand J Infect Dis. 2003;35(10):713-8.

5. Chaban B, Ngeleka M, Hill JE. Detection and quantification of 14 Campylobacter species in pet dogs reveals an increase in species richness in feces of diarrheic animals. BMC Microbiol. 2010;10:73. doi:10.1186/1471-2180-10-73.

6. Parsons BN, Porter CJ, Ryvar R, Stavisky J, Williams NJ, Pinchbeck GL, et al. Prevalence of Campylobacter spp. in a cross-sectional study of dogs attending veterinary practices in the UK and risk indicators associated with shedding. Veterinary J. 2010;184(1):66-70. doi:http://dx.doi.org/10.1016/j. tvil.2009.01.009.

7. Weese JS. Bacterial Enteritis in Dogs and Cats: Diagnosis, Therapy, and Zoonotic Potential. Vet Clin North Am Small Anim Pract. 2011;41(2):287-309.

8. Sandberg M, Bergsjo B, Hofshagen M, Skjerve E, Kruse H. Risk factors for Campylobacter infection in Norwegian cats and dogs. Prev Vet Med. 2002;55(4):241-53.

9. Acke E, McGill K, Golden O, Jones BR, Fanning S, Whyte P. Prevalence of thermophilic Campylobacter species in household cats and dogs in Ireland. Vet Rec. 2009;164(2):44-7

10. Workman SN, Mathison GE, Lavoie MC. Pet dogs and chicken meat as reservoirs of Campylobacter spp. in Barbados. J Clin Microbiol. 2005;43(6):2642-50. doi:10.1128/JCM.43.6.2642-2650.2005.

11. Sandstedt K, Wierup M. Concomitant occurrence of Campylobacter and parvoviruses in dogs with gastroenteritis. Vet Res Commun. 1981;4(4):271-3.

12. Leonard EK, Pearl DL, Janecko N, Weese JS, Reid-Smith RJ, Peregrine AS, et al. Factors related to Campylobacter spp. carriage in client-owned dogs visiting veterinary clinics in a region of Ontario, Canada. Epidemiology \& Infection. 2011;139(Special Issue 10):1531-41. doi:doi:10.1017/ S0950268810002906

13. Man SM. The clinical importance of emerging Campylobacter species. Nat Rev Gastroenterol Hepatol. 2011;8(12):669-85.

14. Folkhälsomyndigheten. http://www.folkhalsomyndigheten.se/documents/ statistik-uppfoljning/smittsamma-sjukdomar/2012/brucellos-epidemiologiskarsrapport-2012-2013-101-8.pdf. 2013.

15. Neimann J, Engberg J, Molbak K, Wegener HC. A case-control study of risk factors for sporadic campylobacter infections in Denmark. Epidemiol Infect. 2003;130(3):353-66.

16. Studahl A, Andersson Y. Risk factors for indigenous campylobacter infection: a Swedish case-control study. Epidemiol Infect. 2000;125(2):269-75.

17. Carrique-Mas J, Andersson Y, Hjertqvist M, Svensson A, Torner A, Giesecke J. Risk factors for domestic sporadic campylobacteriosis among young children in Sweden. Scand J Infect Dis. 2005;37(2):101-10. doi:10.1080/00365540510027165.

18. Damborg P, Olsen KE, Moller Nielsen E, Guardabassi L. Occurrence of Campylobacter jejuni in pets living with human patients infected with C. jejuni. J Clin Microbiol. 2004;42(3):1363-4.

19. Wolfs TF, Duim B, Geelen SP, Rigter A, Thomson-Carter F, Fleer A, et al. Neonatal sepsis by Campylobacter jejuni: genetically proven transmission from a household puppy. Clin Infect Dis. 2001;32(5):E97-9. doi:10.1086/319224.

20. Tenkate TD, Stafford RJ. Risk factors for campylobacter infection in infants and young children: a matched case-control study. Epidemiol Infect. 2001;127(3):399-404. 
21. Gras LM, Smid JH, Wagenaar JA, Koene MG, Havelaar AH, Friesema $\mathbb{H}$ et al. Increased risk for Campylobacter jejuni and C. coli infection of pet origin in dog owners and evidence for genetic association between strains causing infection in humans and their pets. Epidemiol Infect. 2013:1-10. doi:10.1017/S0950268813000356.

22. Carbonero A, Torralbo A, Borge C, Garcia-Bocanegra I, Arenas A, Perea A. Campylobacter spp., C. jejuni and C. upsaliensis infection-associated factors in healthy and ill dogs from clinics in Cordoba, Spain. Screening tests for antimicrobial susceptibility. Comp Immunol Microbiol Infect Dis. 2012;35(6):505-12. doi:10.1016/j.cimid.2012.05.002.

23. Bessede E, Solecki O, Sifre E, Labadi L, Megraud F. Identification of Campylobacter species and related organisms by matrix assisted laser desorption ionization-time of flight (MALDI-TOF) mass spectrometry. Clin Microbiol Infect. 2011;17(11):1735-9. doi:10.1111/j.1469-0691.2011.03468.x.

24. Lawson AJ, Linton D, Stanley J, Owen RJ. Polymerase chain reaction detection and speciation of Campylobacter upsaliensis and C. helveticus in human faeces and comparison with culture techniques. J Appl Microbiol. 1997;83(3):375-80.

25. Denis M, Soumet C, Rivoal K, Ermel G, Blivet D, Salvat G, et al. Development of a m-PCR assay for simultaneous identification of Campylobacter jejuni and C. coli. Lett Appl Microbiol. 1999;29(6):406-10.

26. Dingle KE, Colles FM, Wareing DR, Ure R, Fox AJ, Bolton FE, et al. Multilocus sequence typing system for Campylobacter jejuni. J Clin Microbiol. 2001;39(1):14-23. doi:10.1128/JCM. 39.1.14-23.2001.

27. Procter TD, Pearl DL, Finley RL, Leonard EK, Janecko N, Reid-Smith RJ, et al. A Cross-Sectional study examining Campylobacter and other zoonotic enteric pathogens in dogs that frequent dog parks in three cities in South-Western Ontario and risk factors for shedding of Campylobacter spp. Zoonoses Public Health. 2013. doi:10.1111/zph.12062.

28. Parsons BN, Williams NJ, Pinchbeck GL, Christley RM, Hart CA, Gaskell RM, et al. Prevalence and shedding patterns of Campylobacter spp. in longitudinal studies of kennelled dogs. Vet J. 2011;190(2):249-54. doi:10.1016/j.tvjl.2010.10.006.

29. Wieland B, Regula G, Danuser J, Wittwer M, Burnens AP, Wassenaar TM, et al. Campylobacter spp. in dogs and cats in Switzerland: Risk factor analysis and molecular characterization with AFLP. J Vet Med Ser B-Infect Dis Vet Public Health. 2005;52(4):183-9. doi:10.1111/j.1439-0450.2005.00843.x.

30. Amar C, Kittl S, Spreng D, Thomann A, Korczak BM, Burnens AP, et al. Genotypes and antibiotic resistance of canine Campylobacter jejuni isolates. Vet Microbiol. 2014;168(1):124-30.

31. ECDC, Annual Epidemiological Report 2012. Reporting on 2010 surveillance data and 2011 epidemic intelligence data. Stockholm: ECDC; 2013.

32. Patton CM, Shaffer N, Edmonds P, Barrett TJ, Lambert MA, Baker C, et al. Human disease associated with "Campylobacter upsaliensis" (catalasenegative or weakly positive Campylobacter species) in the United States. J Clin Microbiol. 1989;27(1):66-73.

33. Taylor DE, Hiratsuka K, Mueller L. Isolation and characterization of catalasenegative and catalase-weak strains of Campylobacter species, including "Campylobacter upsaliensis," from humans with gastroenteritis. J Clin Microbiol. 1989;27(9):2042-5.

34. Labarca JA, Sturgeon J, Borenstein L, Salem N, Harvey SM, Lehnkering E, et al. Campylobacter upsaliensis: Another pathogen for consideration in the United States. Clin Infect Dis. 2002;34(11):E59-60. doi:10.1086/340266.

35. Goossens H, Giesendorf BA, Vandamme P, Vlaes L, Van den Borre C, Koeken $A$, et al. Investigation of an outbreak of Campylobacter upsaliensis in day care centers in Brussels: analysis of relationships among isolates by phenotypic and genotypic typing methods. J Infect Dis. 1995;172(5):1298-305.

36. Damborg P, Guardabassi L, Pedersen K, Kokotovic B. Comparative analysis of human and canine Campylobacter upsaliensis isolates by amplified fragment length polymorphism. J Clin Microbiol. 2008;46(4):1504-6. doi:10.1128/JCM. 00079-08.

37. Westgarth C, Porter CJ, Nicolson L, Birtles RJ, Williams NJ, Hart CA, et al. Risk factors for the carriage of Campylobacter upsaliensis by dogs in a community in Cheshire. Vet Rec. 2009;165(18):526-30.

38. Manning G, Dowson CG, Bagnall MC, Ahmed IH, West M, Newell DG. Multilocus sequence typing for comparison of veterinary and human isolates of Campylobacter jejuni. Appl Environ Microbiol. 2003;69(11):6370-9.

\section{Submit your next manuscript to BioMed Central and take full advantage of:}

- Convenient online submission

- Thorough peer review

- No space constraints or color figure charges

- Immediate publication on acceptance

- Inclusion in PubMed, CAS, Scopus and Google Scholar

- Research which is freely available for redistribution

Submit your manuscript at www.biomedcentral.com/submit 